\title{
Prophylaxis and Management of Skin Toxicities
}

\author{
Martin Salzmann $^{a, b} \quad$ Frederik Marmé $^{a, c}$ Jessica C. Hassel ${ }^{a, b}$ \\ ${ }^{a}$ National Center for Tumor Diseases, University Hospital Heidelberg, Heidelberg, ${ }^{b}$ Department of Dermatology, \\ University Hospital Heidelberg, Heidelberg, and 'Department of Obstetrics and Gynecology, University Hospital \\ Heidelberg, Heidelberg, Germany
}

\section{Keywords}

Skin toxicity · Targeted therapy · Chemotherapy

\begin{abstract}
Skin toxicity is a common problem not only when treating breast cancer but in all cancer types. Visible on the surface, these side effects come not just with burdening symptoms but also with stigmatization. With increasing diversity in therapeutic options, dermatologic side effects are also becoming increasingly complex and more challenging for the clinician. We reviewed the most common dermatologic side effects of current anticancer therapy, including toxicity induced by chemotherapy, targeted therapy, and immunotherapy. In particular, we focus on xerosis and pruritus, (acneiform) exanthema, hand-foot syndrome, nail toxicities, alopecia, and mucositis. We propose measures for the prevention and management of these side effects based on current literature. With high incidences in both chemotherapy and targeted therapy, prophylactic measures are crucial to reducing the incidence and severity of skin toxicity and therefore key to therapy adherence. Appropriate management of these toxicities will help avoid unnecessary treatment discontinuation.
\end{abstract}

\section{Introduction}

Cancer therapies can result in a wide variety of dermatologic toxicities affecting the skin, oral mucosa, hair, and nails. Such skin toxicities have been a long-standing problem in the treatment of breast cancer. Some of the most widely used chemotherapies, e.g., capecitabine, taxanes and pegylated liposomal doxorubicin, cause significant skin reactions. The incidence and severity of these dermatologic toxicities are major contributors to the common practice of using lower starting doses, e.g., for capecitabine.

Treatment of breast cancer is becoming increasingly complex with the introduction of a growing number of new targeted therapies associated with an increasing variety of dermatologic toxicities. Some of these are characteristic for the inhibition of the respective signaling pathway, like the acneiform rash caused by epidermal growth factor receptor (EGFR) inhibition (e.g., by lapatinib, a dual HER1/HER2 tyrosine kinase inhibitor (TKI)) or the oral mucositis associated with inhibition of the PI3K/ AKT/mTOR pathway (e.g., by the approved mTOR inhibitor everolimus or novel agents such as PIK3CA inhibitors (e.g., alpelisib) and AKT inhibitors (e.g., ipatasertib) which have recently demonstrated promising results in randomized trials). Many of these agents can cause additional less characteristic skin toxicities. The imminent approval of immune checkpoint inhibitors for the treatment of metastatic breast cancer will add a new layer to the complexity of treatment-associated skin toxicities. Xerosis and pruritus are common problems, but these agents can also cause other skin reactions including psoriatic lesions, lichenoid dermatitis, or sarcoidosis. Rarely, severe cutaneous immune-related adverse events like bullous pemphigoid or even life-threatening StevensJohnson syndrome can occur.

\section{KARGER}

(C) 2019 S. Karger AG, Basel 
This article focusses on the prevention and management of the most common dermatologic toxicities associated with drugs commonly used in the treatment of breast cancer or novel drugs about to enter clinical practice.

\section{Xerosis and Pruritus}

Xerosis and pruritus are widely underrepresented side effects of many anticancer therapies, yet they have shown to reduce health-related quality of life more than most other toxicities [1]. The cause to these symptoms is not always found to be drug-related, xerotic skin is prevalent in healthy subjects also, especially in the elderly. However, symptoms can be induced by numerous medications, especially by various anticancer therapies. Pruritus can occur with or without visible skin changes; in most cases, there are no visible skin lesions other than xerosis. Xerosis and pruritus are most prevalent in EGFR inhibitor therapy, which may be due to inhibition of the EGFR in keratinocytes and sebostasis. Studies report xerosis rates of $7-90 \%$ and pruritus rates of $15-60 \%$ under EGFR inhibitors, with increasing numbers in long-term therapy of up to $100 \%$ for both xerosis and pruritus in patients treated at least 6 months [2]. However, pruritus is common with most targeted anticancer drugs at $13 \%$ for BcrAbl inhibitors, $9-19 \%$ for multikinase inhibitors, and $24 \%$ for mTOR inhibitors. Checkpoint inhibitors such as ipilimumab and the PD-1 antibodies can induce pruritus in $18-34 \%$ [3]. For all of them, grade 3 pruritus is rare $(<3 \%)$. Possible skin alterations include excoriations from scratching or prurigo nodules. Rash or exanthema may also go along with prurigo. Xerosis may come with desquamation of the skin or rhagades. In clinically difficult cases, a dermatologic evaluation should be performed to rule out other underlying causes, e.g., bullous pemphigoid.

\section{Prophylaxis}

Many cases of pruritus can be avoided by diligent skin care with creams and lotions containing 5-10\% urea twice daily. Ultraviolet (UV) radiation exposure should be avoided, as well as extensive washing and other stress to the skin (mechanical, heat, humidity, occlusion).

\section{Management}

Skin care with the above mentioned creams is central to therapy. For mild pruritus, this is the treatment of choice; if indicated, oral antihistamine drugs can be added. For moderate cases, skin care and oral histamines such as cetirizine are always indicated; topical class 2 steroid treatment (e.g., prednicarbate cream) can be added for inflamed skin. In severe cases, all of the above should be used and the patient should be referred to a derma- tologist to optimize the treatment. A pilot study showed promising results in severe pruritus for the off-label use of aprepitant [4].

\section{Exanthema/Rash}

Drug-induced exanthema is the most commonly reported drug reaction by any type of drug, often induced by e.g. antibiotics or non-steroidal anti-inflammatory drugs such as ibuprofen. Typically, maculopapular exanthemas are widespread across the whole body, often starting off at the trunk and disseminating to the arms and legs, and regularly lead to treatment discontinuation. Classical exanthema, however, is a T-cell-mediated effect and not specific to anticancer therapy. It can occur with numerous other medications also. With EGFR and MEK inhibitors, acneiform papulopustular exanthema is a common and expected side effect that is pathogenetically different. Acneiform rashes are typically distributed along the seborrheic areas of the skin, including the face, neck, and upper trunk. They can be aggravated by sunlight. Overall, $40-90 \%$ of patients develop this side effect with a higher frequency in patients under 50 years [5]; it is most common in patients treated with cetuximab and panitumumab (70-90\%) and less common but still prevalent with lapatinib (28-45\%), and by trend more severe with afatinib (16\%) $[6,7] .57 \%$ of patients receiving a MEK inhibitor will develop an acneiform rash [8] (fig. 1a). In contrast, exanthemas induced by BRAF inhibitors are mostly maculopapular and occur in between 14 and $40 \%$ of patients if treatment is combined with a MEK inhibitor (fig. 1b) [9]. They seem to be dose-dependent and can be aggravated by UV light. For treatment with immune checkpoint inhibitors, mild exanthema represents the most common immune-related adverse event [3]. To grade the severity of the rash, typically the Common Terminology Criteria for Adverse Events (CTCAE) are used which are based on the effected body surface area. However, as most exanthemas cover the whole body, it is more the appearance of the lesions including confluence, grade of inflammation, and symptoms which make an exanthema severe. Severe exanthemas can be accompanied by fever, chills, pain, and edema.

\section{Prophylaxis}

Most exanthemas cannot be prevented as they are classical side effects of the drug. However, consistent skin care as well as avoiding sunlight might be of support. Especially under targeted treatments such as EGFR, BRAF, and MEK inhibitors, UV protection avoids aggravation of the exanthema. As acneiform exanthemas under EGFR inhibitor therapy are so frequent, prophylactic use of oral tetracyclines is recommended. Several studies have inves- 
Fig. 1. a EGFR antibody(panitumumab)-induced acneiform exanthema; b BRAF/MEK inhibitor(vemurafenib + cobimetinib)-induced maculopapular exanthema.

Fig. 2. Chemotherapy(doxorubicin)-induced hand-foot syndrome type 1 on a hands, $\mathbf{b}$ feet, and $\mathbf{c}$ the axillaries.
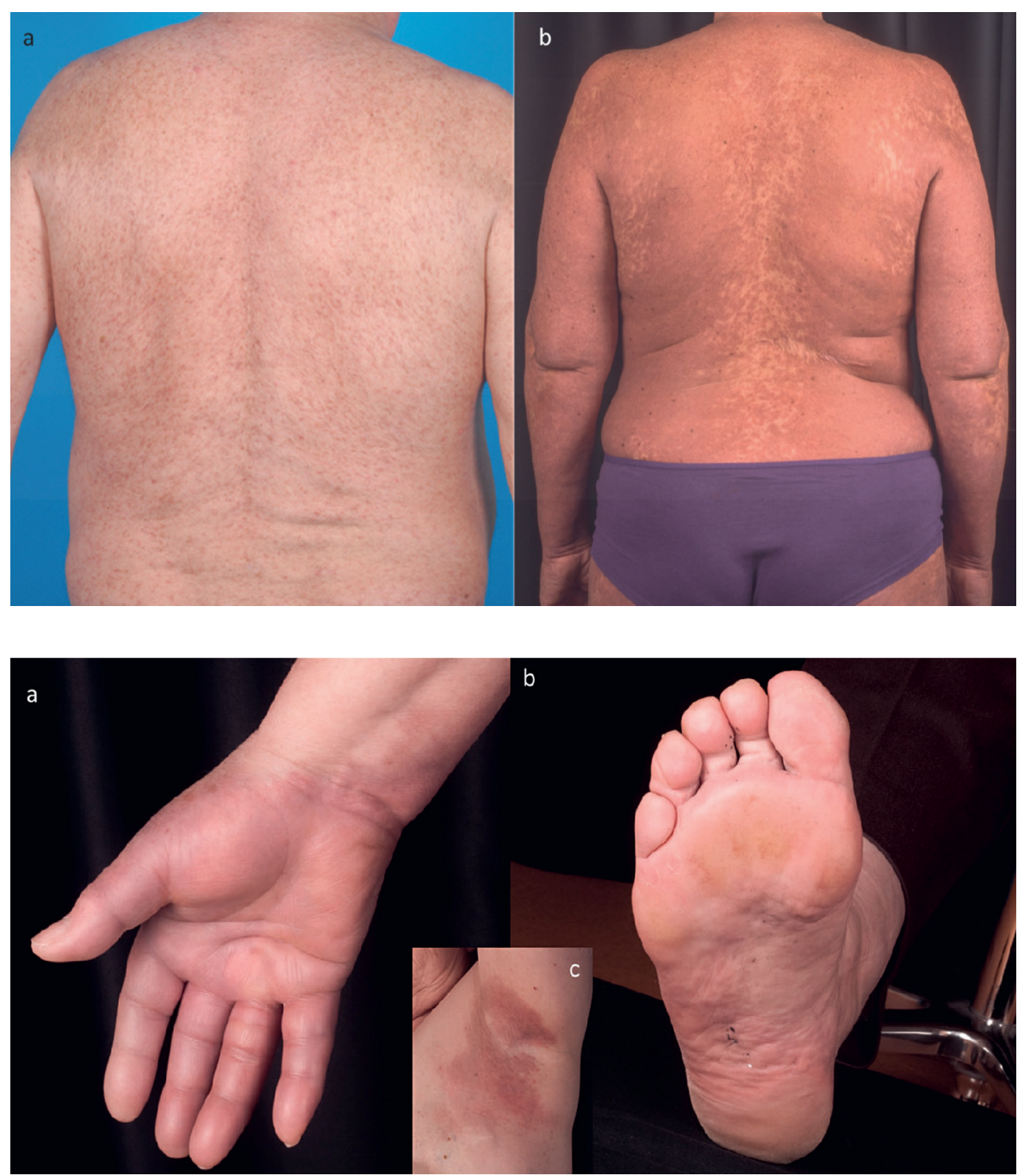

tigated the effect of prophylactic doxycycline $100 \mathrm{mg}$ twice daily and minocycline $50 \mathrm{mg}$ twice daily. Prophylactic treatment reduced the severity of the rash; however, the overall incidence was unchanged [10].

\section{Management}

The treatment of a drug-induced rash should be based on its severity. For mild exanthemas, moisturizing ointments might be sufficient and can be combined with oral antihistamines if pruritic. For acneiform rashes, topical antibiotics can be applied, e.g., metronidazole $1 \%$ or erythromycin $2 \%$ cream. In more inflamed moderate exanthemas, topical steroids should be added, e.g., mometasone ointment. In most mild cases, the anticancer treatment can be continued. If the skin is severely affected, a dermatologic evaluation should be sought to rule out other skin diseases and obtain a management recommendation. Also, dose modifications and even interruption or discontinuation of the anticancer treatment might be needed. Systemic steroid treatment may be used to quickly reduce inflammation.

\section{Hand-Foot Syndrome}

Hand-foot syndrome (HFS) is defined by painful, erythematous palmoplantar lesions that may occur under various anticancer treatments. It is thought to be a collection of diseases of different pathogenesis leading to a heterogeneous spectrum of clinical presentations. The current understanding divides the disease into 2 types: Type 1 is common in chemotherapy and may occur early (1-21 days) in high-dose therapy but also late (several months) in low-dose therapy. Pathogenetically, symptoms might be explained by excretion of the drug with the sweat. It is most common with pyrimidine analogues (e.g., 5-fluorouracil (5-FU), capecitabine, cytarabine), taxanes (e.g., docetaxel), and anthracyclines (e.g., doxorubicin) but may also occur less frequently in various other chemotherapies. Incidence correlates with chemotherapy dose and combination [11]. Type 1 HFS typically presents with painful bullous erythema, edema, and desquamation (fig. 2). Type 2 HFS is common in targeted therapy, mostly in multikinase inhibitors as well as BRAF inhibitors, 
with highest frequencies in sorafenib (up to 48\%) and sunitinib (up to $36 \%$ ); compared to chemotherapy, grade 3 is more common (17 and $23 \%$, respectively [12]). Lesions usually present as callus-like hyperkeratoses or bullae that may be accompanied by an erythematous halo. Lesions are more localized, especially to areas exposed to mechanical pressure.

\section{Prevention}

It is commonly accepted to search for and treat already existing diseases of the hands and feet, such as mycosis and hyperkeratosis, before the start of therapy. Patients should apply lotions and creams containing 5-10\% urea at least twice daily and reduce physical stress to the skin such as heat, pressure, rubbing, occlusion, and contact with chemicals. Cooling of the hands and feet, e.g., by using a cooling glove during the administration of chemotherapy (in particular docetaxel), can be tried to reduce blood flow to the extremities.

\section{Management}

There are no controlled trials regarding the treatment of HFS. It is commonly accepted to continue all the above prophylactic measures. Also, topical steroids should be added (class 2-3, e.g., prednicarbate or mometasone cream). In HFS grade 3, chemotherapy or targeted therapy should be paused until grade $0-1$ is reached. In the case of reccurrence that cannot be controlled by topical treatment, dose reduction or discontinuation should be evaluated.

\section{Nail Toxicities}

Nail toxicities may present in various forms with both chemotherapy and targeted therapy. They are most likely to occur with taxanes and EGFR-targeted therapy [13]. Different treatment regimens cause different types of nail toxicities varying from melanonychia to leukonychia and onycholysis. Cyclophosphamide, for example, causes a typical diffuse black proximal and longitudinal discoloration, doxorubicin causes alternating bands of brown and white lines, and taxanes can lead to orange discolorations caused by hemorrhages. Typical white lines are caused by doxorubicin, cyclophosphamide, and vincristine and represent each chemotherapy cycle. Especially with taxanes, onycholysis is common. Various types of chemotherapy as well as EGFR antagonists cause periungual lesions, mostly paronychia. Onycholysis, onychomadesis, periungual lesions, as well as onychoschizia, onychorrhexis, hematomas, abscesses, or subungual granulomas may be associated with pain, especially under mechanical pressure. Nail toxicities might be so severe that they significantly impact daily life. Regrowth of the nail is expect- ed after discontinuation, which may, however, go along with persisting deformities or dysesthesia.

With docetaxel, a prospective study found the incidence of nail toxicities to be $26 \%$, with $11 \%$ grade 3 [14]. In EGFR-targeted therapy, heterogeneous percentages are reported to range from 4 to $67 \%$; grade 3 is rare [15].

\section{Prevention}

Patients should keep their nails short and avoid mechanical stress to the fingers and feet, such as pressure, humidity, tight shoes, contact with chemicals, but also nail manipulation. Manicure should be done with care. There are no systematic studies investigating these recommendations. There is evidence for cooling of the hands and feet during docetaxel infusion which is thus recommended [16]. The use of oral biotin (off-label) is not evidence-based but common. Some patients benefit from local application of urea-containing nail polish if the nails are thin and tend to break.

\section{Management}

All preventive measures should be continued or intensified. This particularly applies to chemotherapy-induced onychodystrophia, onycholysis, and paronychia. Melanonychia or leukonychia usually do not need to be treated and can be covered up by nail coloring. Paronychia induced by EGFR-targeted or chemotherapy is prone to superinfection, which should be treated by daily use of antiseptic baths and creams. In severe cases, systemic antibiotics or surgery are indicated $[6,17]$.

\section{Alopecia}

Alopecia is the most common side effect of chemotherapy (and some targeted therapies) and among the most psychologically impairing. Patients may refuse therapy due to stigmatization [18]. Chemotherapy-induced alopecia is an anagen-dystrophic effluvium with hair being destroyed in the active growth phase. High rates occur with paclitaxel $(>80 \%)$, doxorubicin $(60-$ $100 \%$ ), and cyclophosphamide (>60\%). Rates are highest in polychemotherapy [19]. In targeted therapy, a meta-analysis revealed overall incidence rates of $15 \%$ with the highest incidence for the hedgehog inhibitor vismodegib (57\%) which often leads to complete hair loss. However, the risk of alopecia is lower than in chemotherapy [20].

\section{Prevention}

Drug treatment has not shown any success in the prevention of alopecia. Different studies suggest scalp cooling to be an effective way to prevent alopecia [21]. The COOLHAIR study, a randomized trial in patients treated 
with taxanes or anthracyclines, showed significant prevention of hair loss by scalp cooling [22]. Other prophylactic measures have had very limited success and are not recommended.

\section{Management}

There is no known therapy for alopecia. Informing the patient about the expected hair loss and about artificial hair and giving the patient early access to these alternatives is crucial to avoid stigmatization.

\section{Mucositis}

Oral mucositis is a common side effect of chemotherapy. The pathogenesis is believed to be a complex mechanism of damage to DNA, upregulation of proinflammatory cytokines and enzymes, apoptosis, ulceration, as well as secondary infection [23]. With novel therapies, oral toxicities may present in various different ways [24], including, but not limited to, dysgeusia, aphthoid lesions, mucosal bleeding, xerostomia, glossitis, lichenoid reactions, as well as hyperkeratosis and gingival hyperplasia. Secondary squamous cell carcinoma is a rare complication in chronic inflammation and patients should be monitored; persisting ulcerations might need to be biopsied. For chemotherapy, the incidence of mucositis is increased with combinations of different cytostatic drugs. Highest rates are reported for anthracyclines + docetaxel + 5-FU (66\%), docetaxel + 5-FU (46\%), and docetaxel + 5 -FU + platinum (43\%). For most other combinations as well as most monotherapies, rates of $10-20 \%$ are common [25]. Lack of oral care, xerostomia, genetic factors, renal/hepatic insufficiency, and previous antitumor therapy of the head and neck area are known to increase the risk of mucositis [23]. Symptoms may range from painless erythema to ulceration, bleeding, and pain. Mucositis is most common on the inside of the lips and cheeks, lateral and ventral tongue, and soft palate. Fungal superinfection can be a relevant problem, as well as a higher incidence of febrile neutropenia which may occur simultaneously.

\section{Prevention}

Preventive measures include consistent care of the mucosa by moisturization of the mucosa, dental care using a soft toothbrush and floss, and avoiding hot and spicy foods as well as tobacco, alcohol, and fluids containing a high amount of sugar or acid. Regular examinations should be performed by a dentist and the treating oncologist. Also, there is evidence for mouth cooling being of benefit, e.g., by sucking an ice cube during therapy [26], as well as the use of dexamethasone mouth wash 4 times daily for patients treated with everolimus [27]. The use of many other supportive measures such as honey, chewing gum, or chamomile is common but not evidence-based and hence not recommended.

\section{Therapy}

All of the above preventive measures should be continued or intensified. Mouth wash with doxepin 5\% (off-label) or lidocaine can decrease pain, also systemic pain medication should be given as needed. There is no treatment for the underlying cause of mucositis other than discontinuation of therapy, which may be necessary in severe cases.

\section{Conclusion}

Skin toxicities are common in nearly all anticancer therapies. Knowing the most common side effects and related preventive measures and management is crucial to avoiding discontinuation of therapy due to skin toxicity. In severe cases, dermatologic advice is recommended to optimize patient treatment. The topic of cutaneous side effects will be of utmost importance going forward, with particular importance directed towards immune checkpoint inhibitors which are associated with a broad range of cutaneous immune-related adverse events such as lichenoid dermatitis, psoriatic lesions, or sarcoidosis.

\section{Funding Sources}

None.

\section{Author Contributions}

All authors contributed written content to this article.

\section{Statement of Ethics}

The authors have no ethical conflicts to disclose.

\section{Disclosure Statement}

MS has received honoraria and travel reimbursements from Bristol-Myers Squibb (BMS), Novartis, Pfizer, Merck, and Abbvie. FM has received honoraria, travel grants, and research funding from AstraZeneca, Tesaro, Roche, Novartis, Pfizer, PharmaMar, GenomicHealth, CureVac, Amgen, Eisai, MSD, GenomicHealth, Clovis, and Celgene. JCH receives honoraria for talks from BMS, MSD, Novartis, Roche, and Pfizer and was a consultant in an advisory board for Pierre Fabre and Sanofi. 


\section{References}

-1 Gandhi M, Oishi K, Zubal B, Lacouture ME: Unanticipated toxicities from anticancer therapies: survivors' perspectives. Support Care Cancer 2010;18:1461-1468.

-2 Osio A, Mateus C, Soria JC, Massard C, Malka D, Boige V, Besse B, Robert C: Cutaneous side-effects in patients on long-term treatment with epidermal growth factor receptor inhibitors. Br J Dermatol 2009;161:515-521.

$\checkmark 3$ Hassel JC, Heinzerling L, Aberle J, Bahr O, Eigentler TK, Grimm MO, Grunwald V, Leipe J, Reinmuth N, Tietze JK, Trojan J, Zimmer L, Gutzmer R: Combined immune checkpoint blockade (anti-PD-1/anti-CTLA-4): evaluation and management of adverse drug reactions. Cancer Treat Rev 2017;57:36-49.

4 Santini D, Vincenzi B, Guida FM, Imperatori M, Schiavon G, Venditti O, Frezza AM, Berti P, Tonini G: Aprepitant for management of severe pruritus related to biological cancer treatments: a pilot study. Lancet Oncol 2012; 13:1020-1024.

5 Azim HA Jr, Agbor-Tarh D, Bradbury I, Dinh P, Baselga J, Di Cosimo S, Greger JG Jr, Smith I, Jackisch C, Kim SB, Aktas B, Huang CS, Vuylsteke P, Hsieh RK, Dreosti L, Eidtmann H, Piccart M, de Azambuja E: Pattern of rash, diarrhea, and hepatic toxicities secondary to lapatinib and their association with age and response to neoadjuvant therapy: analysis from the NeoALTTO trial. J Clin Oncol 2013; 31:4504-4511.

6 Potthoff KM, Hassel JC, Wollenberg A, Hofheinz R-D: Therapie und Prophylaxe EGFRinhibitorinduzierter Hautreaktionen. Arzneimitteltherapie 2010;28:191-198.

>7 Yang JC, Wu YL, Schuler M, et al: Afatinib versus cisplatin-based chemotherapy for EGFR mutation-positive lung adenocarcinoma (LUX-Lung 3 and LUX-Lung 6): analysis of overall survival data from two randomised, phase 3 trials. Lancet Oncol 2015;16:141-151.

$\checkmark 8$ Grimaldi AM, Simeone E, Festino L, Vanella V, Strudel M, Ascierto PA: MEK inhibitors in the treatment of metastatic melanoma and solid tumors. Am J Clin Dermatol 2017;18: 745-754.

-9 Flaherty KT, Infante JR, Daud A, et al: Combined BRAF and MEK inhibition in melanoma with BRAF v600 mutations. N Engl J Med 2012;367:1694-1703.

10 Melosky B, Anderson H, Burkes RL, Chu Q, Hao D, Ho V, Ho C, Lam W, Lee CW, Leighl NB, Murray N, Sun S, Winston R, Laskin JJ:
Pan Canadian Rash Trial: a randomized phase III trial evaluating the impact of a prophylactic skin treatment regimen on epidermal growth factor receptor-tyrosine kinase inhibitor-induced skin toxicities in patients with metastatic lung cancer. J Clin Oncol 2016;34: 810-815.

11 Heo YS, Chang HM, Kim TW, Ryu MH, Ahn JH, Kim SB, Lee JS, Kim WK, Cho HK, Kang YK: Hand-foot syndrome in patients treated with capecitabine-containing combination chemotherapy. J Clin Pharmacol 2004;44: 1166-1172.

-12 Lee WJ, Lee JL, Chang SE, Lee MW, Kang YK, Choi JH, Moon KC, Koh JK: Cutaneous adverse effects in patients treated with the multitargeted kinase inhibitors sorafenib and sunitinib. Br J Dermatol 2009;161:1045-1051.

13 Robert C, Sibaud V, Mateus C, Verschoore M, Charles C, Lanoy E, Baran R: Nail toxicities induced by systemic anticancer treatments. Lancet Oncol 2015; 16:e181-189.

14 Hong J, Park SH, Choi SJ, Lee SH, Lee KC, Lee JI, Kyung SY, An CH, Lee SP, Park JW, Jeong SH, Nam E, Bang SM, Cho EK, Shin DB, Lee JH: Nail toxicity after treatment with docetaxel: a prospective analysis in patients with advanced non-small cell lung cancer. Jpn J Clin Oncol 2007;37:424-428.

15 Kozuki T: Skin problems and EGFR-tyrosine kinase inhibitor. Jpn J Clin Oncol 2016;46: 291-298.

16 Scotte F, Banu E, Medioni J, Levy E, Ebenezer C, Marsan S, Banu A, Tourani JM, Andrieu JM, Oudard S: Matched case-control phase 2 study to evaluate the use of a frozen sock to prevent docetaxel-induced onycholysis and cutaneous toxicity of the foot. Cancer 2008; 112:1625-1631.

17 Lacouture ME, Anadkat MJ, Bensadoun RJ, Bryce J, Chan A, Epstein JB, Eaby-Sandy B, Murphy BA: Clinical practice guidelines for the prevention and treatment of EGFR inhibitor-associated dermatologic toxicities. Support Care Cancer 2011;19:1079-1095.

18 Hackbarth M, Haas N, Fotopoulou C, Lichtenegger W, Sehouli J: Chemotherapy-induced dermatological toxicity: frequencies and impact on quality of life in women's cancers. Results of a prospective study. Support Care Cancer 2008;16:267-273.

19 Trueb RM: Chemotherapy-induced alopecia. Curr Opin Support Palliat Care 2010;4:281284.
20 Belum VR, Marulanda K, Ensslin C, Gorcey L, Parikh T, Wu S, Busam KJ, Gerber PA, Lacouture ME: Alopecia in patients treated with molecularly targeted anticancer therapies. Ann Oncol 2015;26:2496-2502.

21 Shin H, Jo SJ, Kim DH, Kwon O, Myung SK: Efficacy of interventions for prevention of chemotherapy-induced alopecia: a systematic review and meta-analysis. Int J Cancer 2015; 136:E442-E454.

22 Smetanay K, Junio P, Feisst M, Seitz J, Hassel JC, Mayer L, Matthies LM, Schumann A, Hennigs A, Heil J, Sohn C, Jaeger D, Schneeweiss A, Marme F: COOLHAIR: a prospective randomized trial to investigate the efficacy and tolerability of scalp cooling in patients undergoing (neo)adjuvant chemotherapy for early breast cancer. Breast Cancer Res Treat 2018;DOI: 10.1007/s10549018-4983-8.

23 Al-Dasooqi N, Sonis ST, Bowen JM, Bateman E, Blijlevens N, Gibson RJ, Logan RM, Nair RG, Stringer AM, Yazbeck R, Elad S, Lalla RV: Emerging evidence on the pathobiology of mucositis. Support Care Cancer 2013;21: 3233-3241.

24 Vigarios E, Epstein JB, Sibaud V: Oral mucosal changes induced by anticancer targeted therapies and immune checkpoint inhibitors. Support Care Cancer 2017;25:1713-1739.

25 Sonis ST, Elting LS, Keefe D, Peterson DE, Schubert M, Hauer-Jensen M, Bekele BN, Raber-Durlacher J, Donnelly JP, Rubenstein EB: Perspectives on cancer therapy-induced mucosal injury: pathogenesis, measurement, epidemiology, and consequences for patients. Cancer 2004;100:1995-2025.

26 Lalla RV, Bowen J, Barasch A, Elting L, Epstein J, Keefe DM, McGuire DB, Migliorati C, Nicolatou-Galitis O, Peterson DE, RaberDurlacher JE, Sonis ST, Elad S: MASCC/ ISOO clinical practice guidelines for the management of mucositis secondary to cancer therapy. Cancer 2014;120:1453-1461.

-27 Rugo HS, Seneviratne L, Beck JT, Glaspy JA, Peguero JA, Pluard TJ, Dhillon N, Hwang LC, Nangia C, Mayer IA, Meiller TF, Chambers MS, Sweetman RW, Sabo JR, Litton JK: Prevention of everolimus-related stomatitis in women with hormone receptor-positive, HER2-negative metastatic breast cancer using dexamethasone mouthwash (SWISH): a single-arm, phase 2 trial. Lancet Oncol 2017; 18:654-662. 